\title{
Localisation et restitution des sources vibratoires : une approche numérique et expérimentale
}

\author{
Xavier Chiementin ${ }^{1, a}$, Fabrice Bolaers ${ }^{1}$, Lanto Rasolofondraibe ${ }^{2}$ \\ Et Jean-Paul Dron ${ }^{1}$ \\ 1 GMMS, Groupe de Mécanique, Matériaux et Structures, Université de Reims, BP 1039, 51687 Reims Cedex 2, France \\ 2 CReSTIC, Centre de Recherche en Sciences et Technologies de l'Information et de la Communication, Université de Reims, \\ BP 1039, 51687 Reims Cedex 2, France
}

Reçu le 19 novembre 2005, accepté le 7 décembre 2006

\begin{abstract}
Résumé - L'analyse vibratoire permet d'appréhender l'état de fonctionnement de machines tournantes. Le signal, enregistré par des accéléromètres, peut contenir, simultanément, des informations de plusieurs composants rendant difficile l'interprétation de l'état de l'un d'entre eux. Dans ce cadre les problèmes inverses s'avèrent être un outil intéressant. Ils permettent de revenir à l'information originale à partir d'observations. Leurs instabilités nécessitent un grand nombre d'observations face au nombre de sources, et demandent l'emploi de méthodes dites de régularisation. Cependant une régularisation signifie une perte d'information, et l'emploi d'un système surdéterminé entraîne une qualité de l'information moindre. Cet article propose de pallier à ces problèmes en optimisant la position des capteurs de vibration afin de rendre l'inversion la plus exacte possible. Cette optimisation sera obtenue d'une part par une approche numérique, qui nécessite un recalage du modèle, et d'autre part par une approche expérimentale, grâce à l'analyse modale.
\end{abstract}

Mots clés : Sources vibratoires / problème inverse / analyse modale

\begin{abstract}
Localization and restitution of vibratory sources: a numerical and experimental approach. The vibratory analysis makes it possible to apprehend the operating condition revolving machines. The signal, recorded by accelerometers, can contain, at the same time, information of several components making difficult the interpretation of the state of them. Within this framework inverse problems prove to be an interesting tool. They make it possible to return to original information starting from observations. Their instabilities require large number of observations facing the number of sources, and the use of method known as of regularization. However a regularization means loss of information and the use of a surdetermined system causes a basic quality of information. This paper proposes to avoid these problems by optimizing the position of vibration sensor in order to make the inversion most exactly possible. This optimization will be obtained on the one hand by an approach numerical, requiring an updating model, and on the other hand by an experimental approach, thanks to the modal analysis.
\end{abstract}

Key words: Vibratory sources / inverse problem / modal analysis

\section{Introduction}

De nombreux domaines exigent de retrouver les contributions d'origines à partir d'observations et la connaissance du milieu de propagation [1]. Les problèmes inverses répondent à cette demande. La littérature montre la diversité des domaines d'application : mécanique,

\footnotetext{
a Auteur correspondant :

xavier.chiementin@univ-reims.fr
}

sismographie, biologie [2]. Leur implémentation dans le cadre de l'analyse vibratoire des machines de production serait un atout majeur. En effet, l'arrêt d'une production causé par une panne mécanique peut s'avérer contraignant aussi bien pour la productivité que pour la protection du personnel. C'est pour ces deux raisons que la maintenance conditionnelle par analyse vibratoire est devenue l'un des piliers majeurs de l'industrie [3]. Elle permet, à partir d'outils d'analyse, de diagnostiquer des défauts des composants tournants 


\section{Nomenclature}

\begin{tabular}{|c|c|}
\hline \multicolumn{2}{|c|}{ Exposants } \\
\hline$T$ & Transposée \\
\hline$*$ & Conjuguée \\
\hline$H$ & Transposée conjuguée \\
\hline+ & Inverse \\
\hline \multicolumn{2}{|c|}{ Notations } \\
\hline$\|\ldots\|_{1}$ & Norme 1 \\
\hline$\|\ldots\|_{2}$ & Norme 2 \\
\hline$E R$ & Erreur relative \\
\hline$x_{i}$ & Entrée/Source $i$ \\
\hline$y_{i}$ & Sortie/Capteur $i$ \\
\hline$c(\mathbf{H})$ & Nombre de conditionnement de la matrice $\mathbf{H}$ \\
\hline$\Gamma_{x x}$ & Matrice spectrale des signaux entrées \\
\hline$\Gamma_{y y}$ & Matrice spectrale des signaux sorties \\
\hline$h_{i \rightarrow j}$ & Fonction de transfert entre le point $i$ et $j$ \\
\hline$t_{i \rightarrow j}$ & Fonction de transmissibilité entre le point $i$ et $j$ \\
\hline$\vec{Y}$ & Vecteurs sorties \\
\hline$\vec{X}$ & Vecteurs entrées \\
\hline $\mathbf{H}$ & Matrice de transfert \\
\hline $\mathbf{T}$ & Matrice de transmissibilité \\
\hline M & Matrice de masse exprimée dans la base modale \\
\hline $\mathbf{C}$ & Matrice d'amortissement exprimée dans la base modale \\
\hline $\mathbf{K}$ & Matrice de raideur exprimée dans la base modale \\
\hline$\phi_{m}$ & Vecteur propre du mode $m$ \\
\hline$\omega$ & Pulsation rad.s ${ }^{-1}$ \\
\hline$\sigma_{i}$ & $i^{\text {ème }}$ valeur singulière \\
\hline$E R_{x}$ & Erreur relative sur l'entrée \\
\hline$E R_{y}$ & Erreur relative sur la sortie \\
\hline$\delta \vec{X}$ & Variation sur les données entrées \\
\hline$\delta \vec{Y}$ & Variation sur les données sorties \\
\hline$\delta \mathbf{H}$ & Variation sur les données de la matrice de mélange \\
\hline
\end{tabular}

sensibles tels que les roulements. Les informations sont collectées, le plus souvent, par un capteur de vibration type piézoélectrique. Celles-ci sont analysées par diverses méthodes afin d'appréhender l'état de fonctionnement de la machine [4-6] puis de suivre l'état d'endommagement [7]. Un état de l'art sur ce sujet est réalisé par Tandon [8]. Pour utiliser au mieux ces méthodes, il est préférable de placer le capteur au plus près du composant à étudier. Cependant l'accès au palier du roulement n'est pas toujours possible, c'est pourquoi il est nécessaire de placer les capteurs en un autre point [9]. Le capteur ainsi positionné peut porter l'information de plusieurs composants. Ce constat peut s'avérer très gênant si ces composants sont identiques, menant à une erreur de diagnostic certain car la séparation des informations est impossible. C'est pourquoi les problèmes inverses peuvent prétendre restituer les informations générées par chaque composant.
La structure mécanique étant connue, il est possible de localiser les roulements considérés comme étant les sources vibratoires. Certaines méthodes proposent de réaliser des inversions sans connaissance de la matrice de mélange, en supposant l'indépendance statistique des entrées. Cependant ces méthodes semblent peu adaptées dans le cadre de la localisation de défauts [10], car d'une part la solution d'une inversion n'est pas unique et d'autre part les entrées ne sont en général pas statisquement indépendantes. L'ensemble des études réalisées avec les techniques de problèmes inverses cherche à restituer l'ensemble du signal vibratoire provenant des sources [11-14], donnant lieu à des méthodes de régularisation. De plus elles proposent une restitution réalisée à partir de deux voire trois fois plus de capteurs que de sources. L'originalité de ce travail est de ne considérer que des bandes de fréquences qui correspondent aux fréquences 
caractéristiques de défauts potentiels sur une structure, et d'utiliser un nombre limité de capteurs par rapport aux sources. Ainsi il est possible de réaliser une restitution énergétique de chaque fréquence caractéristique des défauts présents, en positionnant de manière adéquate les capteurs sur la structure. Cet article présente les méthodes inverses, leurs applications et leurs limites. Cette présentation permettra de proposer une méthode de restitution par un positionnement optimal des capteurs de vibration. Cette optimisation sera réalisée par deux approches qui seront correlées au préalable : une approche numérique, introduisant les cartes de conditionnement [15], et une approche expérimentale liée à l'analyse modale d'une structure.

\section{Problèmes inverses}

\subsection{Description}

Les problèmes inverses permettent de retrouver les contributions d'origines à partir d'observations (Fig. 1). Ils permettent entre autres de traiter les systèmes linéaires modélisés par la relation équation (1) :

$$
\vec{Y}=\mathbf{H} \cdot \vec{X}
$$

Les vecteurs $\vec{Y}$ et $\vec{X}$ représentent respectivement les vecteurs des sorties et les vecteurs des entrées tandis que $\mathbf{H}$ illustre la matrice de mélange. Pour une étude vibratoire, telle qu'elle est menée dans cet article, le mélange est convolutif, équation (2) :

$$
y_{i}(t)=\Sigma_{j=1}^{p} h_{i, j}(t) * x_{j}(t)
$$

où $p$ est le nombre d'entrées et $m$ le nombre de sorties. $h_{i, j}$ représente la réponse impulsionnelle du milieu entre la sortie $j$ et l'entrée $i$. Le passage dans le domaine fréquentiel permet d'obtenir une relation linéaire, équation (1). Nous avons utilisé la méthode d'inversion de la matrice de mélange basée sur la décomposition en valeurs singulières, équation (3) :

$$
\mathbf{H}^{+}=\mathbf{V} \cdot \Sigma^{+} \cdot \mathbf{U}^{t}
$$

où $\mathbf{U}$ et $\mathbf{V}$ sont les matrices unitaires de type $(p, p)$ et $(m, m), \Sigma$ est une matrice diagonale dont les éléments sont les valeurs singulières $\sigma_{i}$ de la matrice $\mathbf{H}$, équation (4). [ ] ${ }^{+}$représente l'inverse de la matrice

$$
\Sigma^{+}=\left(\begin{array}{cccc}
\frac{1}{\sigma_{1}} & 0 & \ldots & 0 \\
0 & \frac{1}{\sigma_{2}} & \ldots & \\
\vdots & & \ddots & \vdots \\
0 & & 0 & \frac{1}{\sigma_{r}}
\end{array}\right)
$$

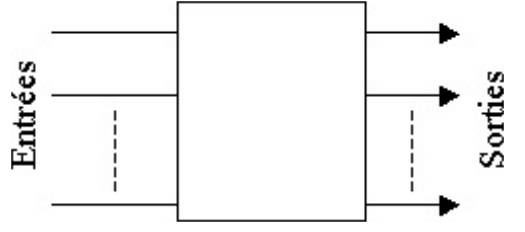

Fig. 1. Système MIMO.

\subsection{Contraintes expérimentales et numériques des méthodes d'inversion}

En général les méthodes d'inversion sont des problèmes mal posés. Un modèle bien posé répond à trois critères [16] : l'unicité, l'existence et la stabilité de la solution. La troisième hypothèse n'est que rarement vérifiée. En effet, des petites erreurs de mesures peuvent donner lieu à d'importantes erreurs sur les entrées calculées. Les chaînes d'acquisition, les mesures expérimentales, les parasites sont autant de paramètres qui rendent le problème mal posé. Les perturbations engendrées par des erreurs de mesures sont notées $[\delta \mathbf{H}]$ et/ou $[\delta \vec{Y}]$, l'introduction de ces erreurs dans l'équation (1) nous donne la relation équation (5):

$$
\vec{Y}+\delta \vec{Y}=(\mathbf{H}+\delta \mathbf{H})(\vec{X}+\delta \vec{X})
$$

Dans le cas où la matrice peut être inversée on peut écrire l'inéquation (6) :

$$
\frac{\|\delta \vec{X}\|_{1}}{\|\vec{X}\|_{1}} \leq M \cdot c(\mathbf{H}) \cdot\left(\frac{\|\delta \mathbf{H}\|_{1}}{\|\mathbf{H}\|_{1}}+\frac{\|\delta \vec{Y}\|_{1}}{\|\vec{Y}\|_{1}}\right)
$$

avec

$$
M=\frac{1}{1-\left\|(\delta \mathbf{H}) \cdot \mathbf{H}^{-1}\right\|_{1}} \quad \text { et } \quad c(\mathbf{H})=\|\mathbf{H}\|_{2} \cdot\left\|\mathbf{H}^{-1}\right\|_{2}
$$

\| $\|_{i}$ représente la norme $i . c(\mathbf{H})$ est le nombre de conditionnement de $\mathbf{H}$. Dans la pratique, les erreurs de mesures sont faibles donc $M$ est proche de l'unité. Ainsi l'effet des erreurs de mesures sur la solution dépend principalement du conditionnement de la matrice de transfert [17]. Une matrice dont le nombre de conditionnement est élevé sera dite mal conditionnée, tandis que s'il est petit, elle sera dite bien conditionnée. Les erreurs commises sur le vecteur sorties et la matrice de mélange vont être transmises vers la matrice des entrées. D'après Zhang [17], les relations (8) et (9) définissent le domaine d'erreur :

$$
\begin{gathered}
\frac{1}{c^{2}(\mathbf{H})} E R_{x} \leq E R_{y} \leq c^{2}(\mathbf{H}) E R_{x} \\
E R_{x} \leq \frac{1}{\sigma_{\min }^{2}} .\|\delta(\mathbf{H})\|_{2}^{2}
\end{gathered}
$$

$E R_{x}$ et $E R_{y}$ représentent respectivement les erreurs relatives des entrées et des sorties. Le nombre de conditionnement $c(\mathbf{H})$ doit être le plus petit possible pour que l'erreur ne puisse pas s'amplifier. 
Pour améliorer la méthode d'inversion, il est nécessaire de prendre quelques précautions.

L'utilisation de l'équation (10) liant la matrice des densités spectrales de sorties $\Gamma_{y y}$ et celle d'entrées $\Gamma_{x x}$ est prescrite. Cette relation basée sur les statistiques a l'avantage d'atténuer le bruit enregistré [12] :

$$
\Gamma_{y y}=\mathbf{H}^{H} \cdot \Gamma_{x x} . \mathbf{H}
$$

où []$^{H}$ représente le conjugué de sa transposée.

Il est préférable d'utiliser un système surdéterminé pour limiter les effets de bruits, c'est-à-dire comprenant plus de sorties que d'entrées. Dans ces conditions le problème est un problème «pseudo-inverse ». Ensuite, pour éviter l'augmentation des erreurs, certaines méthodes, dites de régularisation, existent. La régularisation consiste soit simplement à annuler toutes les valeurs singulières jugées petites soit à utiliser une méthode d'optimisation, qui régularise la plus petite valeur singulière $[2,18]$.

Cependant la régularisation traduit une perte d'informations alors que l'objectif est de restituer au mieux toute l'énergie disponible. De plus, un système surdéterminé ne permet pas une restitution énergétique précise. C'est pourquoi cet article propose, d'une part, l'utilisation d'un système carré, contenant autant d'entrées que de sorties et, d'autre part, deux approches pour l'optimisation du positionnement des sorties afin de parfaire la restitution. Cette optimisation permet d'obtenir un nombre de conditionnement le plus petit possible, avec un système nonsurdéterminé.

\section{Application des méthodes d'inversion dans le cadre de la localisation de sources vibratoires}

\subsection{Position du problème}

L'étude se porte sur un système MIMO (Multiple Input, Multiple Output) $p$ entrées $x_{i}$, et $p$ sorties $y_{i}$, d'équation (11) dans le domaine fréquentiel :

$$
\vec{Y}(\omega)=\mathbf{T}(\omega) \cdot \vec{X}(\omega)
$$

$\vec{Y}(\omega), \vec{X}(\omega)$ et $\mathbf{T}(\omega)$ sont respectivement le vecteur de sorties, le vecteur d'entrées et la matrice de mélange. L'objectif est de rendre l'inversion stable pour retrouver la contribution des entrées. Nous mettons en évidence deux approches qui permettent d'augmenter la qualité de l'inversion, une approche numérique et une approche expérimentale.

\subsection{Qualité de l'inversion et approche numérique}

\subsubsection{Carte de conditionnement}

D'après la section 2.2, la stabilité de l'inversion est d'autant plus stable que le nombre de conditionnement est petit. Or celui-ci dépend de la matrice de mélange, c'està-dire de l'environnement, de la position des entrées et des sorties. C'est pourquoi, l'approche numérique donne lieu à des cartes de la structure représentant les isovaleurs de ces nombres. Ces cartes sont appelées « carte de conditionnement ». Ainsi les zones optimales sont le lieu où le nombre de conditionnement sera le plus faible.

\subsubsection{Mode opératoire de l'approche numérique}

Cette approche nécessite un modèle numérique de la structure étudiée. Une sortie est considérée comme étant une variable tandis que les autres sorties sont disposées sur la structure. La sortie variable balaie l'ensemble des points. Pour chaque point de la structure, le nombre de conditionnement est calculé, d'où la naissance de zones d'optimisation et de la carte de conditionnement. Cette carte, ainsi obtenue, permet de positionner la sortie dans une zone où l'inversion sera stable.

\subsection{Qualité de l'inversion et analyse modale}

Le système MIMO a pour relation l'équation (11) où la matrice de mélange $\mathbf{T}(\omega)$ est explicitée par l'équation (12)

$$
\mathbf{T}=\left(\begin{array}{ccc}
t_{x_{1} \rightarrow y_{1}} & \ldots & t_{x_{p} \rightarrow y_{1}} \\
\vdots & & \vdots \\
t_{x_{1} \rightarrow y_{p}} & \ldots & t_{x_{p} \rightarrow y_{p}}
\end{array}\right)
$$

$t_{x_{i} \rightarrow y_{j}}$ est la fonction de transmissibilité, rapport dans le domaine fréquentiel du signal sortie $y_{j}$ et du signal entrée $x_{i}$. $\mathbf{T}$ représente la matrice de mélange appelée également matrice de transmissibilité. Chaque fonction de transmissibilité est obtenue à partir de la fonction de transfert dite classique, tronquée à $M$ modes, équation (13). Ainsi ces fonctions ont pour relation équation (14) :

$$
h_{i j}(\omega)=\frac{A\left(y_{j}\right)}{F\left(x_{i}\right)}=\sum_{k} \frac{\phi_{k}\left(x_{i}\right) \phi_{k}\left(y_{j}\right)}{-\omega^{2} M_{k}+\mathbf{i} \omega C_{k}+K_{k}}
$$

$h_{i j}$ est la fonction de transfert entre les points $x_{i}$ et $y_{j}$. $\phi_{m}(i)$ est la composante du vecteur propre du $m^{\text {ème }}$ mode correspondant au point $i$. $A\left(y_{j}\right)$ et $F\left(x_{i}\right)$ sont respectivement l'accélération au point $y_{j}$ et la force appliquée au point $x_{i} . M_{m}, K_{m}$ et $C_{m}$ sont respectivement les $m^{\text {ème }}$ composantes de la diagonale des matrices de masse, de raideur, et d'amortissement exprimées dans la base modale [19]

$$
\begin{aligned}
t_{x_{i} \rightarrow y_{j}}(\omega) & =\frac{A\left(y_{j}\right)}{A\left(x_{i}\right)}=\frac{h_{i j}(\omega)}{h_{i i}(\omega)}=\frac{A\left(y_{j}\right)}{F\left(x_{i}\right)} \cdot \frac{F\left(x_{i}\right)}{A\left(x_{i}\right)} \\
& =\frac{\sum_{k} \frac{\phi_{k}\left(x_{i}\right) \phi_{k}\left(y_{j}\right)}{-\omega^{2} M_{k}+\mathbf{i} \omega C_{k}+K_{k}}}{\sum_{k} \frac{\phi_{k}\left(x_{i}\right) \phi_{k}\left(x_{i}\right)}{-\omega^{2} M_{k}+\mathbf{i} \omega C_{k}+K_{k}}}
\end{aligned}
$$


Ces fonctions constituent la matrice de mélange. L'inversion de la matrice $\mathbf{T}$ est d'autant plus facile que le nombre de conditionnement est petit ou le déterminant grand. Or ce nombre dépend des fonctions de transmissibilité qui dépendent elles-mêmes de la position des entrées, des sorties et de la fréquence étudiée. Cependant, l'entrée est considérée fixe et l'étude est réalisée en bande étroite autour des fréquences que l'on souhaite restituer. Ceci signifie que la fonction de transmissibilité ne dépend que de la sortie $y_{j}$. Par conséquent, la fonction de transmissibilité devient une combinaison linéaire de $M$ modes, équation (15) :

$$
t_{x_{i} \rightarrow y_{j}}(\omega)=\sum_{k} a_{k}\left(x_{i}, \omega\right) \phi_{k}\left(y_{j}\right)
$$

L'étude de la contribution de chaque terme $a_{k}\left(x_{i}, \omega\right)$ permet de simplifier l'écriture à $M$ termes. Ainsi, les modes qui régissent ces fonctions sont les modes dominants. Le nombre de conditionnement dépend essentiellement des modes dominants de la structure étudiée.

\subsubsection{Cas où un mode domine, deux entrées, deux sorties}

Prenons le cas où $\omega=\omega_{m}, \omega_{m}$ est une résonance de structure. La relation (14) peut s'écrire sous une autre forme (16), mettant en évidence le terme correspondant à la pulsation propre $\omega=\omega_{m}$ :

$t_{x_{i} \rightarrow y_{j}}\left(\omega_{m}\right)=$

$$
\frac{\frac{\phi_{m}\left(x_{i}\right) \phi_{m}\left(y_{j}\right)}{-\omega_{m}^{2} M_{m}+\mathbf{i} \omega_{m} C_{m}+K_{m}}+\sum_{k \neq m} \frac{\phi_{k}\left(x_{i}\right) \phi_{k}\left(y_{j}\right)}{-\omega_{m}^{2} M_{k}+\mathbf{i} \omega_{m} C_{k}+K_{k}}}{\frac{\phi_{m}\left(x_{i}\right) \phi_{m}\left(x_{i}\right)}{-\omega_{m}^{2} M_{m}+\mathbf{i} \omega_{m} C_{m}+K_{m}}+\sum_{k \neq m} \frac{\phi_{k}\left(x_{i}\right) \phi_{k}\left(x_{i}\right)}{-\omega_{m}^{2} M_{k}+\mathbf{i} \omega_{m} C_{k}+K_{k}}}
$$

Dans le cas d'un amortissement faible (cas de structures métalliques), le premier terme du dénominateur et du numérateur ont une contribution dominante. Les termes suivants sont négligeables. Cela signifie que le mode $m$ est représentatif de notre fonction de transmissibilité. On dira qu'un seul mode domine. Ainsi la relation (16) peut être simplifiée suivant (17), ce qui donne la matrice de transmissibilité, équation (18) :

$$
\begin{gathered}
t_{x_{i} \rightarrow y_{j}}=\frac{\phi_{m}\left(y_{j}\right)}{\phi_{m}\left(x_{i}\right)} \\
\mathbf{T}=\left(\begin{array}{ll}
\frac{\phi_{m}\left(y_{1}\right)}{\phi_{m}\left(x_{1}\right)} & \frac{\phi_{m}\left(y_{1}\right)}{\phi_{m}\left(x_{2}\right)} \\
\frac{\phi_{m}\left(y_{2}\right)}{\phi_{m}\left(x_{1}\right)} & \frac{\phi_{m}\left(y_{2}\right)}{\phi_{m}\left(x_{2}\right)}
\end{array}\right)
\end{gathered}
$$

Le déterminant de l'équation (18) est nul. La restitution sera impossible.

\subsubsection{Cas où deux modes dominent, deux entrées, deux sorties}

Les modes $m$ et $n$ dominent chacune des fonctions de transmissibilité donc celles-ci sont la résultante de la combinaison linéaire des vecteurs propres liées aux modes dominants, équation (19) :

$$
t_{x_{i} \rightarrow y_{j}}(\omega)=a_{n}\left(x_{i}, \omega\right) \phi_{n}\left(y_{j}\right)+a_{m}\left(x_{i}, \omega\right) \phi_{m}\left(y_{j}\right)
$$

Le déterminant devient l'equation (20) (cf. Annexe, équation (32) avec $p=2, M=2$ ), où $m$ est le premier mode dominant et $n$ est le second

$$
\operatorname{det}(\mathbf{T})=\frac{1}{\prod_{i=1}^{2} \sum_{k=1}^{2} \frac{\phi_{k}\left(x_{i}\right) \phi_{k}\left(x_{i}\right)}{D_{k}}} \operatorname{det}\left(\mathbf{T}^{\prime}\right)
$$

avec

$$
\begin{aligned}
\operatorname{det}\left(\mathbf{T}^{\prime}\right)= & \frac{1}{D_{1} D_{2}}\left(\phi_{1}\left(x_{1}\right) \phi_{2}\left(x_{2}\right)\right. \\
& \left.-\phi_{1}\left(x_{2}\right) \phi_{2}\left(x_{1}\right)\right)\left(\phi_{1}\left(y_{2}\right) \phi_{2}\left(y_{3}\right)-\phi_{1}\left(y_{3}\right) \phi_{2}\left(y_{2}\right)\right)
\end{aligned}
$$

et

$$
D_{k}=-\omega^{2} M_{k}+\mathbf{i} \omega C_{k}+K_{k}
$$

Ce résultat montre que le déterminant n'est pas toujours nul, mais peut l'être si les modes sont orthogonaux en certains points de la structure. Ceci explique que le nombre de conditionnement est élevé pour certains points et pour certaines fréquences.

Pour que la matrice soit inversible, il est nécessaire de vérifier la relation équation (21) :

$$
\begin{array}{r}
{\left[\phi_{m}\left(x_{2}\right) \phi_{n}\left(x_{1}\right)-\phi_{m}\left(x_{1}\right) \phi_{n}\left(x_{2}\right)\right] \cdot\left[\phi_{m}\left(y_{2}\right) \phi_{n}\left(y_{1}\right)\right.} \\
\left.-\phi_{m}\left(y_{1}\right) \phi_{n}\left(y_{2}\right)\right] \neq 0
\end{array}
$$

Cette relation est symétrique car d'une part il y a un terme correspondant aux entrées et d'autre part un terme correspondant aux sorties. Chacun des termes doit être différent de zéro pour avoir un déterminant non nul.

\subsubsection{Généralisation $M$ modes dominants, $p$ entrées et $p$ sorties}

La généralisation à $M$ modes, $p$ sorties, $p$ entrées, conduit à la relation équation (22), pour $p \geq 2$. 
(cf. Annexe)

$$
\mid \begin{gathered}
\left|\sum_{k_{1}, k_{2}, \ldots, k_{p}=1}^{m} \frac{1}{D_{k_{1}} \ldots D_{k_{p}}} \Phi\left(x, k_{1}, \ldots, k_{p}\right) . \Phi\left(y, k_{1}, \ldots, k_{p}\right)\right| \neq 0, \\
k_{1}<k_{2}<\ldots<k_{p}, \forall i, k_{i} \in \mathbb{N} \\
\text { où } \Phi\left(x, k_{1}, \ldots, k_{p}\right)=\sum_{\substack{i=1 \text { à } p \\
j=0,1}}(-1)^{j} \phi_{k 1}\left(x_{i+(p-1) j}\right) \\
\times \phi_{k 2}\left(x_{i+1+(p-3) j}\right) \ldots \phi_{k p}\left(x_{i+2+(p-2 p+1) j}\right) \\
\text { où } \Phi\left(y, k_{1}, \ldots, k_{p}\right)=\sum_{\substack{i=1 \text { à } p \\
j=0,1}}(-1)^{j} \phi_{k 1}\left(y_{i+(p-1) j}\right) \\
\times \phi_{k 2}\left(y_{i+1+(p-3) j}\right) \ldots \phi_{k p}\left(y_{i+2+(p-2 p+1) j}\right)
\end{gathered}
$$

Les indices des $x$ et $y$ sont modulo $p, p \geq 3$

Le terme $\Phi\left(x, k_{1}, \ldots, k_{p}\right)$ concerne les entré (22) $\Phi\left(y, k_{1}, \ldots, k_{p}\right)$ les sorties.

\subsubsection{Intérêt et mode opératoire de l'approche expérimentale}

Les équations liant le nombre de conditionnement de la matrice de mélange et les modes propres permettent d'appréhender le positionnement des sorties et de limiter leur nombre au nombre d'entrées. Ce principe permet d'éviter les systèmes surdéterminés où l'information d'une des sorties peut nuire à la restitution. De plus il est intéressant de limiter le nombre de sorties, c'est-àdire, le nombre de capteurs car la disposition d'un grand nombre de capteurs est peu viable, économiquement et expérimentalement. Pour une fréquence d'excitation, la structure peut être régie par un ou plusieurs modes propres. Nous avons établi une procédure expérimentale pour déterminer les zones optimales pour le positionnement des capteurs.

- Réaliser l'analyse modale de la structure,

- Déterminer le nombre de modes dominants pour chacune des fréquences que nous souhaitons restituer,

- Sur $n$ capteurs, positionner $(n-1)$ capteurs sur des nœuds de vibrations (nœuds pour lesquels la déformée propre est nulle) pour faciliter la manipulation de la relation équation (22) permettant de placer le $n^{\text {ième }}$ capteur.

\section{Validation numérique et expérimentale}

\subsection{Définitions}

Il est nécessaire, de définir à travers cette section les différentes notions qui seront abordées afin de lever toute ambiguité à travers la lecture.

La restitution est ciblée sur des fréquences caractéristiques pouvant être générées par une excitation périodique (par exemple un défaut de roulement). La
Tableau 1. Caractéristiques de la plaque avant et après recalage.

\begin{tabular}{ccc}
\hline & Avant recalage & Après recalage \\
\hline Longueur $(\mathrm{m})$ & 0,46 & 0,46952 \\
Largeur $(\mathrm{m})$ & 0,4 & 0,4 \\
Épaisseur $(\mathrm{m})$ & 0,005 & 0,00487 \\
$E(\mathrm{~Pa})$ & $7,4 \times 10^{10}$ & 75577447237 \\
Poisson & 0,35 & 0,35 \\
\hline
\end{tabular}

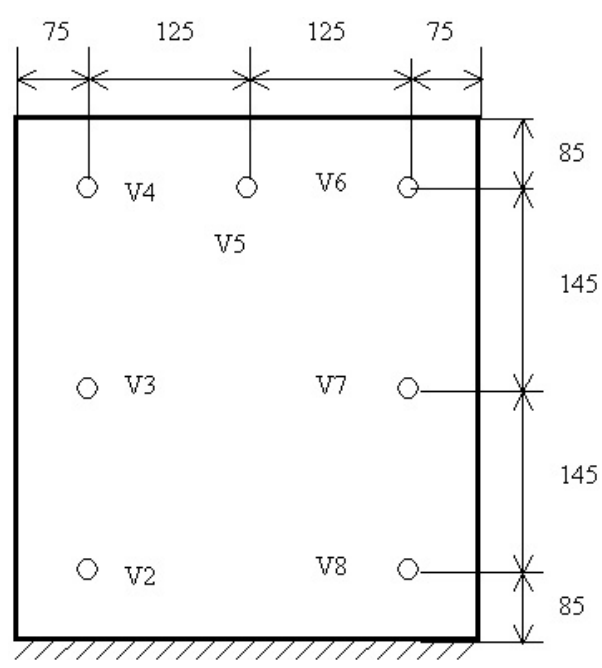

Fig. 2. Position des accéléromètres et du point d'excitation des pots vibrants.

source est un ensemble d'une ou plusieurs fréquences caractéristiques d'un composant mécanique tournant, générée en un point, non contaminée par une autre source ou bruit. Il n'est pas nécessaire que la source soit le point où une force extérieure s'applique. La sortie est un signal vibratoire en un point de la structure, résultant d'un mélange de sources et de bruit. Par conséquent, une 《restitution satisfaisante des sources $»$ signifie que chaque fréquence caractéristique soit restituée à la bonne source. Les entrées (sources) seront notées $x_{i}$ et les sorties (capteurs), $y_{i}$.

\subsection{Description du banc d'expérimentation et recalage du modèle}

L'étude est réalisée sur une plaque en aluminium encastrée libre, dont les propriétés sont répertoriées dans le tableau 1. Elle est encastrée sur un bloc en béton. Les signaux utiles à cette étude sont enregistrés par des accéléromètres piézoélectriques dont les positions sont notées V2 à V8 (Fig. 2). Deux pots vibrants sont fixés par des élastiques et excitent la plaque en deux points distincts, V3 et V7, qui seront les sources (Fig. 3b). Les excitations sont sinusoïdales, de fréquences $70,110 \mathrm{~Hz}$ pour la source 1 et 110, $320 \mathrm{~Hz}$ pour la source 2. Les élastiques permettent d'éviter les modes rigides issus d'une fixation elle-même rigide pouvant perturber les mesures. Les 
Tableau 2. Comparaison des fréquences réelles et numériques après recalage.

\begin{tabular}{cccc}
\hline Modes & $\begin{array}{c}\text { Fréquences réelles } \\
(\mathrm{Hz})\end{array}$ & $\begin{array}{c}\text { Fréquences numériques } \\
\text { après recalage }(\mathrm{Hz})\end{array}$ & $\begin{array}{c}\text { Écart relatif } \\
(\%)\end{array}$ \\
\hline 1 & 18,7 & 19,3 & 1,71 \\
2 & 50,6 & 52,3 & 1,65 \\
3 & 118,7 & 118,5 & 0,08 \\
4 & 181,2 & 184,5 & 0,90 \\
5 & 192,5 & 193,9 & 0,36 \\
6 & 334,4 & 334,2 & 0,03 \\
7 & 355,1 & 354,3 & 0,13 \\
8 & 400,7 & 398,6 & 0,26 \\
9 & 485,6 & 484,5 & 0,11 \\
\hline
\end{tabular}

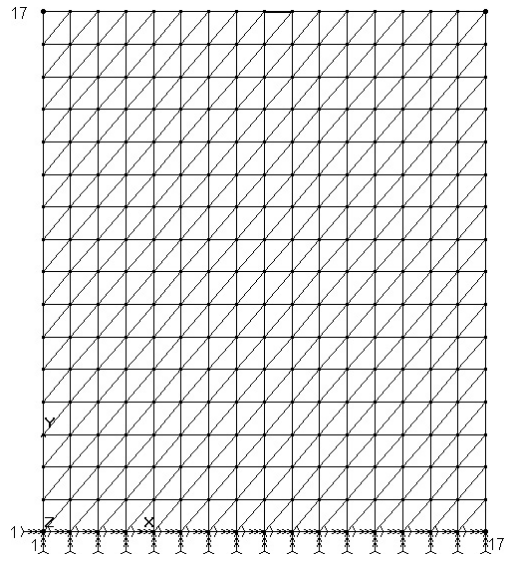

(a)

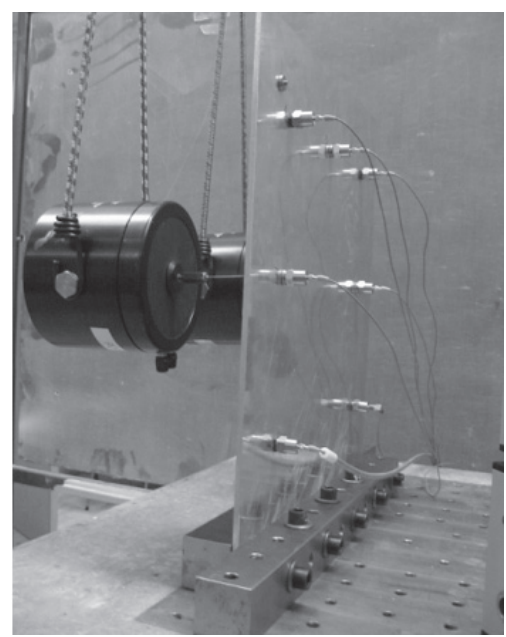

(b)

Fig. 3. (a) Modèle numérique de la plaque encastrée-libre. (b) Banc d'expérimentation.

signaux sont collectés à travers un système d'acquisition 8 voies. Celui-ci permet également de générer des signaux excitateurs qui commandent les pots vibrants. Le modèle numérique a été réalisé à partir d'un logiciel éléments-finis. La plaque est ainsi constituée d'un maillage plan dont le nombre de points s'élève à 289, (Fig. 3a). Le modèle numérique fait l'objet d'un recalage grâce à une boucle minorant la différence quadratique entre les fréquences propres calculées numériquement et déterminées expérimentalement. Le recalage est effectué sur les neuf premières fréquences propres (Tab. 2).

\subsection{Approche numérique}

\subsubsection{Détermination de la carte de conditionnement}

Pour réaliser une optimisation de la position des capteurs dans le cadre de la restitution, la première approche concerne l'utilisation d'un modèle numérique sans prendre en compte la notion d'analyse modale. Un capteur est fixé au point V5 de la structure (Fig. 2). Celuici est choisi de manière à garder une symétrie dans le système et ainsi appréhender au mieux les résultats. Le deuxième capteur balaie tous les points de la structure, faisant varier la matrice de mélange ainsi que le nombre de conditionnement. L'ensemble de ces informations est porté sur une carte donnant lieu à « une carte de conditionnement $»$. Celle-ci représente la plaque avec une représentation en ligne de niveaux des nombres de conditionnement. Ces cartes peuvent être réalisées, soit pour chacune des fréquences (Figs. $4 \mathrm{a}-\mathrm{c}$ ) soit de manière globale, superposant les diverses cartes (Fig. 4d).

Chacune des deux méthodes apporte ses informations. La première méthode permet d'obtenir un critère quant à la qualité de la restitution de chaque fréquence. Si le capteur est placé au point V2, le bon conditionnement de la matrice pour les fréquences 70 et $320 \mathrm{~Hz}$ signifie que la restitution pour ces fréquences sera satisfaisante. Par contre le conditionnement pour la fréquence de $110 \mathrm{~Hz}$ causera l'accentuation des erreurs commises sur les données des capteurs et de la matrice de transmissibilité. La méthode globale nous renseigne directement sur les positions optimales des capteurs afin d'obtenir le plus petit conditionnement possible, sans fournir d'informations sur la restitution des différentes fréquences.

Nous avons constaté que les zones où le conditionnement est inférieur à 20 correspondent à une zone où le 


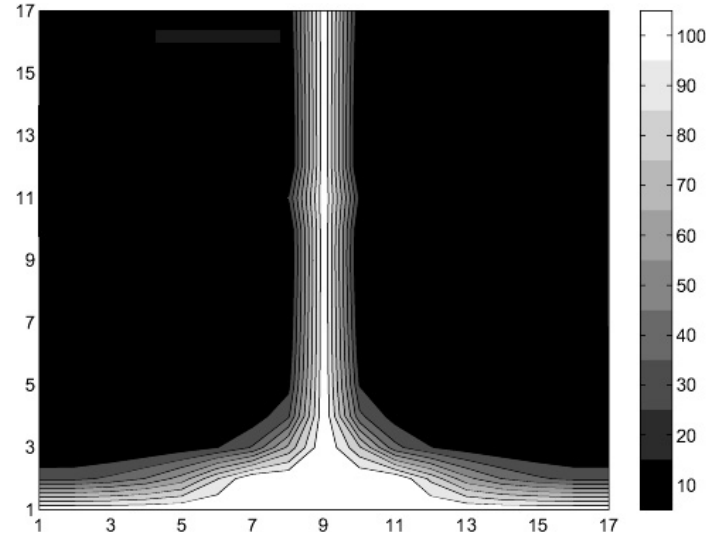

(a)

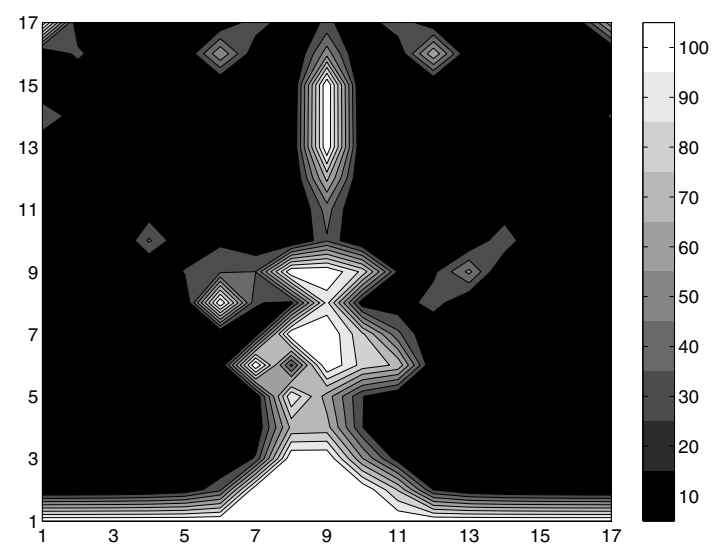

(c)

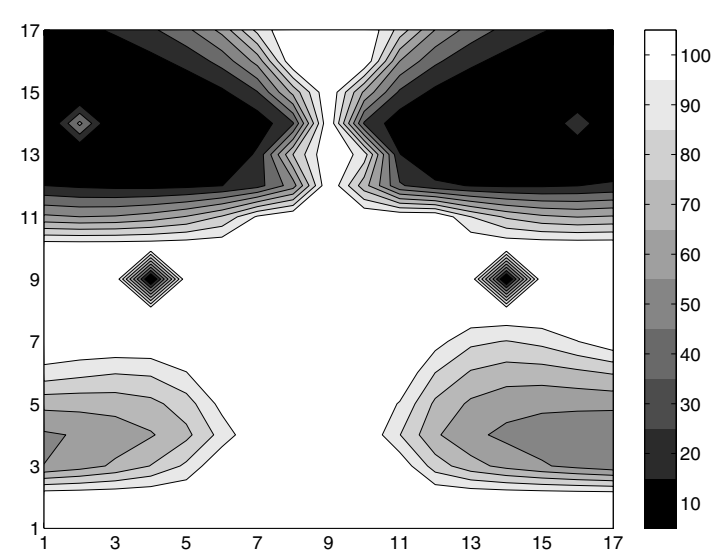

(b)

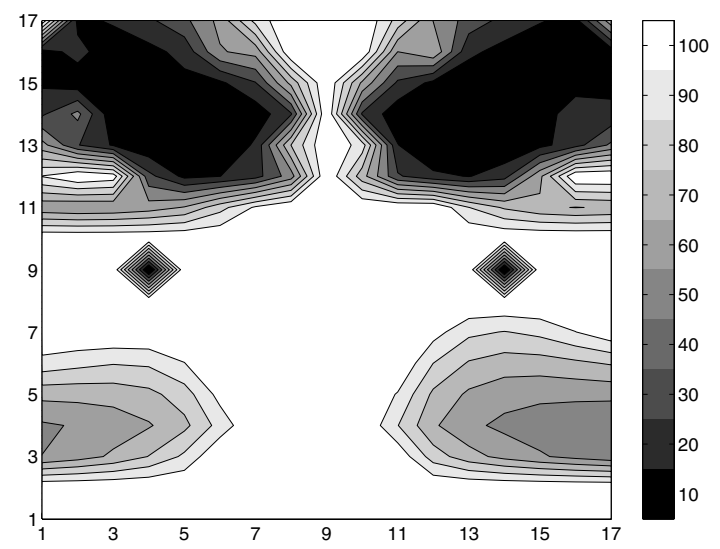

(d)

Fig. 4. Carte de conditionnement, représentant les iso-valeurs des nombres de conditionnement pour (a) $70 \mathrm{~Hz}$, (b) $110 \mathrm{~Hz}$, (c) 320 Hz. (d) Carte globale.

capteur doit être placé, pour obtenir une bonne restitution des sources. Par contre les zones à conditionnement supérieur à ce seuil sont à proscrire.

\subsubsection{Résultats numériques sur la restitution des sources}

Un bruit blanc est introduit dans le système. Nous avons introduit du bruit de l'ordre de $10 \%$ sur les données capteurs. Le capteur mobile est placé successivement aux points V4 et V2, c'est-à-dire placé respectivement dans une zone bien conditionnée et mal conditionnée. Les résultats des inversions (Fig. 5) illustrent la bonne stabilité de la restitution à partir d'une zone bien conditionnée, et par ailleurs une forte instabilité face à un mauvais conditionnement. En effet la restitution présente une perte énergétique de $10 \mathrm{~dB}$ sur la fréquence restituée de $110 \mathrm{~Hz}$ comme prévu par les cartes de conditionnement. La restitution n'est parfaite que si les fréquences à restituer ont une énergie suffisante pour pouvoir les restituer, c'est-à-dire environ dix fois supérieure à l'énergie du bruit. Au-delà de cette valeur la restitution n'est pas satisfaisante.

\subsection{Approche expérimentale}

L'approche expérimentale dans le cadre de la restitution permet d'éviter l'utilisation d'un modèle numérique. Cette approche est basée sur l'analyse modale. À partir du mode opératoire décrit dans la section 3.2, des zones d'optimisation seront déduites pour une fréquence de $70 \mathrm{~Hz}$.

\subsubsection{Recherche des modes dominants}

Dans un premier temps, la recherche des modes dominants est primordiale pour déduire la relation à vérifier. Pour déterminer la contribution de chaque mode, l'écriture de la fonction de transmissibilité est simplifiée en considérant l'entrée fixe. Ainsi la fonction de transmissibilité ne dépend que de $j$, équation (23) :

$$
t_{i \rightarrow j}(j)=\sum_{m} a_{m}(i, \omega) \phi_{m}(j)
$$

Le calcul de la contribution de chaque terme est déduit :

$$
\%_{\text {contribution }}=\frac{a_{p}(i, \omega)}{\sum_{p} a_{p}(i, \omega)}
$$



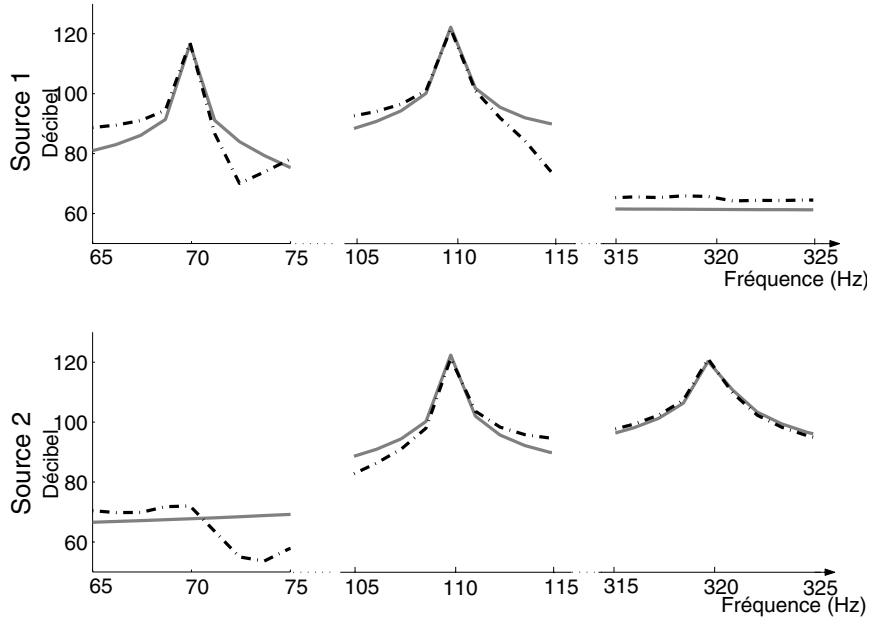

(a)
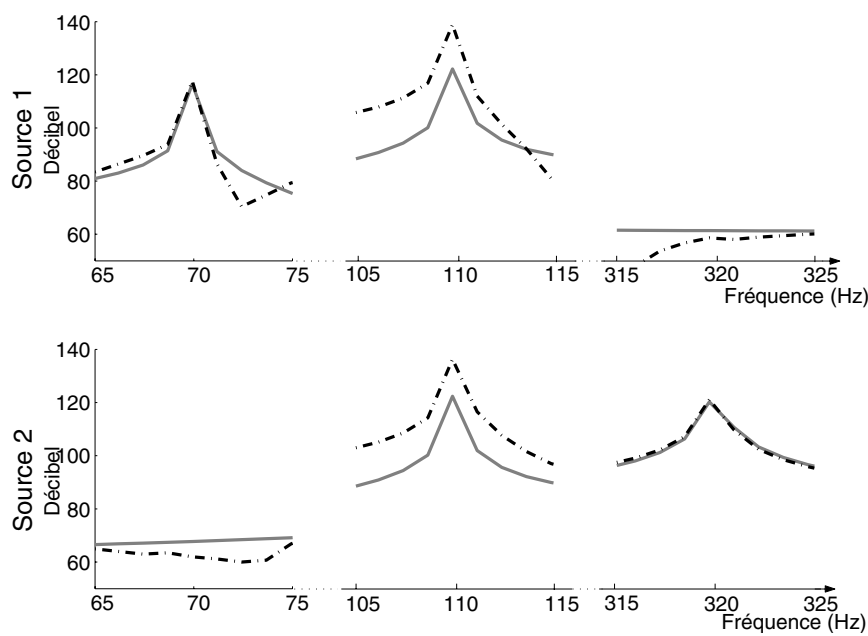

(b)

Fig. 5. Restitution à partir d'une zone (a) bien conditionnée, (b) mal conditionnée. (noir) sources réelles, (gris pointillés) sources calculées par inversion.

L'extraction des modes permet de traiter les informations de l'ensemble des capteurs situés sur la structure. Une excitation sinusoïdale de fréquence $70 \mathrm{~Hz}$ est générée à travers l'un des pots vibrants pour déterminer les fonctions de transmissibilité. Comme le système sourcescapteur 1 est symétrique les fonctions de transmissibilité sont identiques, c'est-à-dire $t_{x_{1} \rightarrow y_{1}}=t_{x_{2} \rightarrow y_{1}}$. Une régression linéaire multiple est réalisée entre les vecteurs propres et chacune des fonctions de transmissibilité afin de définir la contribution de chaque mode pour ces fonctions. Le tableau 3 indique la contribution des 10 premiers modes, et indique que seuls les trois premiers modes interviennent à raison de $92,2 \%$. Il y a donc trois modes dominants.

La connaissance de la forme des déplacements modaux est primordiale pour la suite de la construction. Les trois modes sont illustrés par la figure 6 . Les modes un et trois sont des modes de flexion tandis que le mode deux est un mode de torsion.
Tableau 3. Contribution en $\%$ de chacun des modes.

\begin{tabular}{ccccc}
\hline Mode 1 & Mode 2 & Mode 3 & Mode 4 & Mode 5 \\
19,5 & 51,8 & 20,9 & 6,50 & 0,37 \\
\hline Mode 6 & Mode 7 & Mode 8 & Mode 9 & Mode 10 \\
0,34 & 0,32 & 0,01 & 0,25 & 0,01 \\
\hline
\end{tabular}

Tableau 4. Simplifications dues aux caractéristiques du système.

\begin{tabular}{cc}
\hline Cause & Conséquence \\
\hline Position du capteur 1 & $\phi_{2}\left(y_{1}\right)=0$ \\
Mode 1 & $\phi_{1}\left(x_{1}\right)=\phi_{1}\left(x_{2}\right)$ \\
Mode 2 & $\phi_{2}\left(x_{1}\right)=-\phi_{2}\left(x_{2}\right)$ \\
Mode 3 & $\phi_{3}\left(x_{1}\right)=\phi_{3}\left(x_{2}\right)$ \\
\hline
\end{tabular}

Le problème se résume à un système à deux sources, deux capteurs et trois modes, c'est pourquoi l'équation (25) est à vérifier, (cf. Annexe équation (34) avec $p=2, M=3$ )

$$
\begin{gathered}
\frac{1}{D_{1} D_{2}}\left(\phi_{1}\left(y_{1}\right) \phi_{2}\left(y_{2}\right)-\phi_{1}\left(y_{2}\right) \phi_{2}\left(y_{1}\right)\right)\left(\phi_{1}\left(x_{1}\right) \phi_{2}\left(x_{2}\right)\right. \\
\left.-\phi_{1}\left(x_{2}\right) \phi_{2}\left(x_{1}\right)\right)+\frac{1}{D_{1} D_{3}}\left(\phi_{1}\left(y_{1}\right) \phi_{3}\left(y_{2}\right)\right. \\
\left.-\phi_{1}\left(y_{2}\right) \phi_{3}\left(y_{1}\right)\right)\left(\phi_{1}\left(x_{1}\right) \phi_{3}\left(x_{2}\right)-\phi_{1}\left(x_{2}\right) \phi_{3}\left(x_{1}\right)\right) \\
+\frac{1}{D_{2} D_{3}}\left(\phi_{2}\left(y_{1}\right) \phi_{3}\left(y_{2}\right)-\phi_{2}\left(y_{2}\right) \phi_{3}\left(y_{1}\right)\right)\left(\phi_{2}\left(x_{1}\right) \phi_{3}\left(x_{2}\right)\right. \\
\left.-\phi_{2}\left(x_{2}\right) \phi_{3}\left(x_{1}\right)\right)>\varepsilon
\end{gathered}
$$

La vérification de cette formule va permettre de dégager des zones d'optimisations.

\subsubsection{Mise en évidence de zones d'optimisation}

La connaissance des déformées propres (Fig. 6), et de la position des sources entraîne de nombreuses simplifications de la relation équation (25). Les modes 1 et 3 sont symétriques, donc la déformée de chacun de ces modes sur le point de la première source est identique à celle de la deuxième source. Ces déformées seront inverses pour le deuxième mode. Le choix de la position du capteur 1 n'est pas arbitraire, il est placé en V5 pour avoir une déformée propre nulle. Le tableau 4 décrit les différentes informations que nous apporte la position des sources et du capteur. Ces caractéristiques, injectées dans la relation 


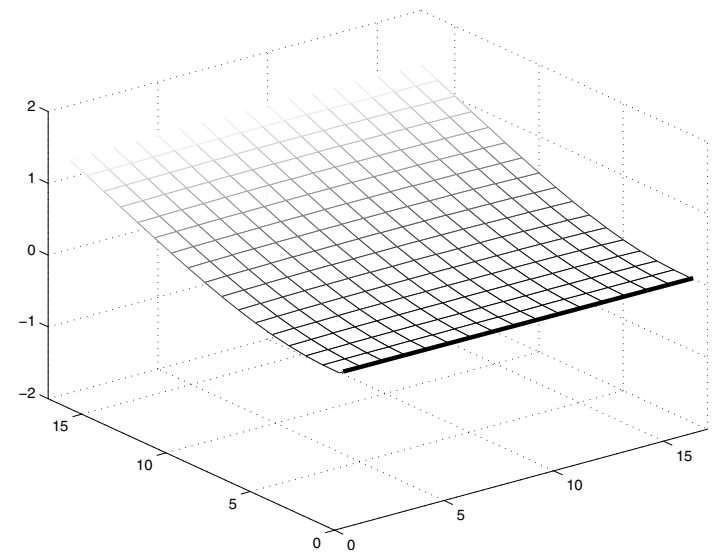

(a)

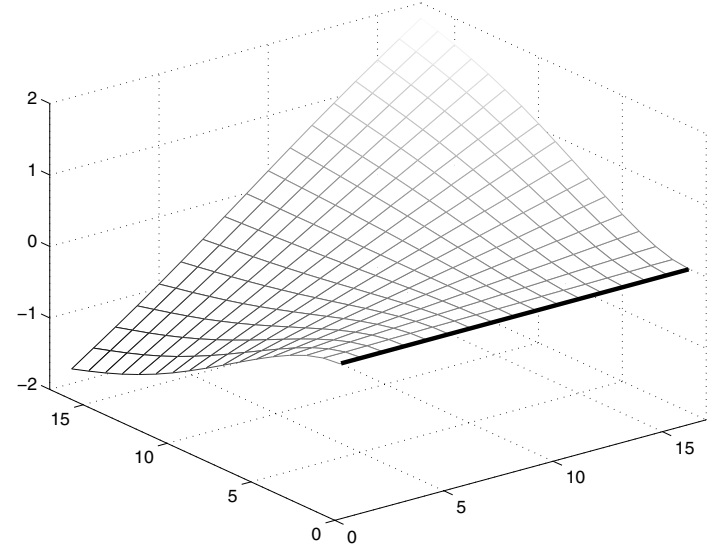

(b)

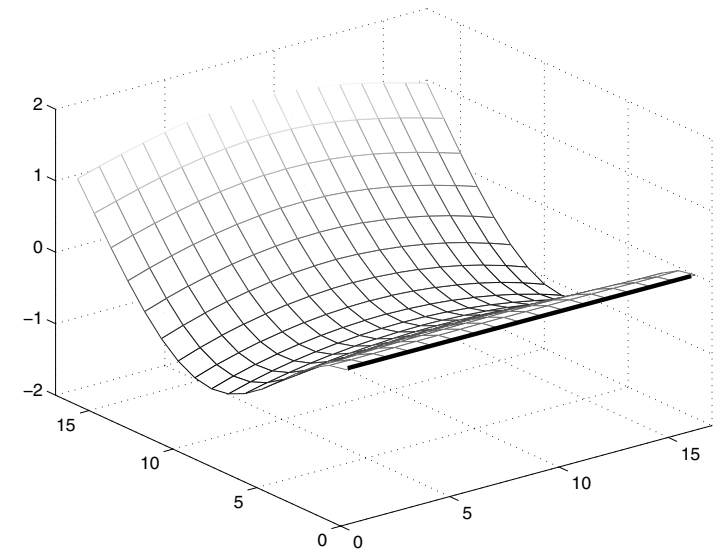

(c)

Fig. 6. (a) Mode 1, (b) Mode 2, (c) Mode 3 de la plaque encastrée-libre.

équation (25), permettent de la simplifier donnant ainsi l'équation (26) :

$$
\begin{aligned}
& \frac{1}{D_{1} D_{2}}\left(\phi_{1}\left(y_{1}\right) \phi_{2}\left(y_{2}\right)\right)\left(2 \phi_{1}\left(x_{1}\right) \phi_{2}\left(x_{2}\right)\right) \\
& \quad+\frac{1}{D_{2} D_{3}}\left(-\phi_{2}\left(y_{2}\right) \phi_{3}\left(y_{1}\right)\right)\left(2 \phi_{2}\left(x_{1}\right) \phi_{3}\left(x_{2}\right)\right)>\varepsilon
\end{aligned}
$$

La seule variable est le déplacement modal du deuxième mode au point du deuxième capteur. Ainsi, mathématiquement, il en ressort que $\phi_{2}\left(y_{2}\right)$ doit être différent de zéro. Ceci signifie que le capteur 2 peut être placé n'importe où sur la plaque sauf sur les nœuds de vibrations (point pour lequel la déformée propre est nulle) du mode 2, soit sur la base de la plaque et sur l'axe central. Une carte de conditionnement peut être déduite (Fig. 7). Celle-ci est très significative de la position optimale du deuxième capteur, et correspond bien à la carte obtenue par l'approche numérique, (Fig. 4a).

Même si la carte est déterminée approximativement, elle reflète les points optimaux. Cependant, sa réalisation est complexe, et paraît difficilement réalisable dans de nombreux cas. Tout d'abord il est nécessaire de connaître le nombre de modes dominants de chaque fonction de transmissibilité composant la matrice de transmissibilité.

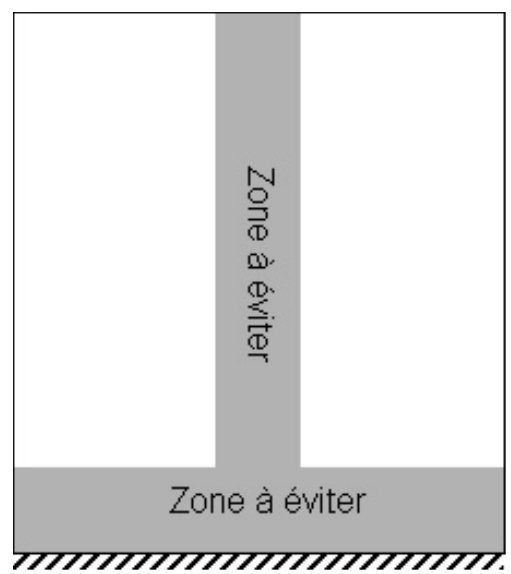

Fig. 7. Carte de conditionnement réalisée par analyse modale.

Par ailleurs, si le nombre de modes dominants est petit, il est possible de déterminer une carte de conditionnement moyennant une bonne disposition des sources. Cependant il est raisonnable de penser, que la relation à vérifier sera d'autant plus complexe que le nombre de modes est important. Ainsi la probabilité qu'elle s'annule diminue. 

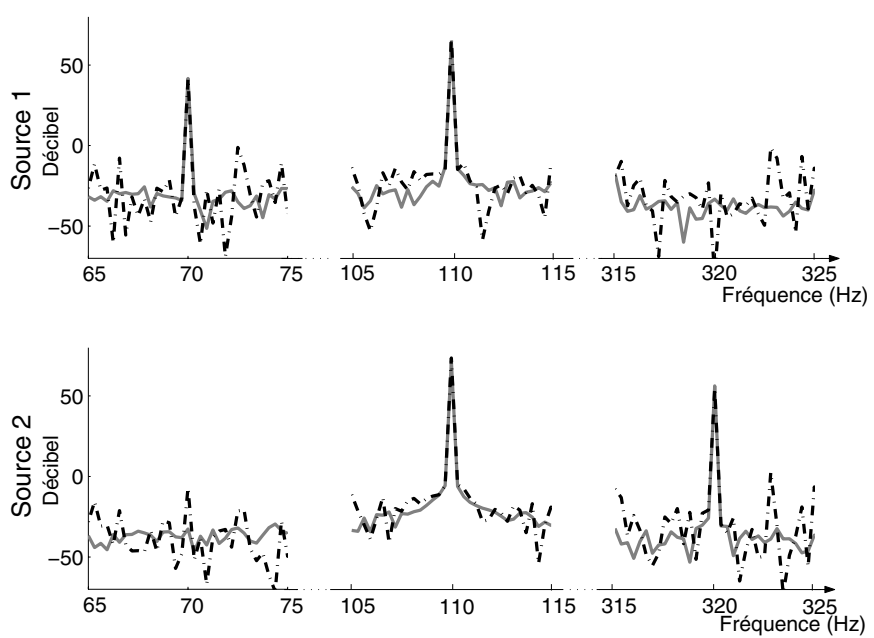

(a)
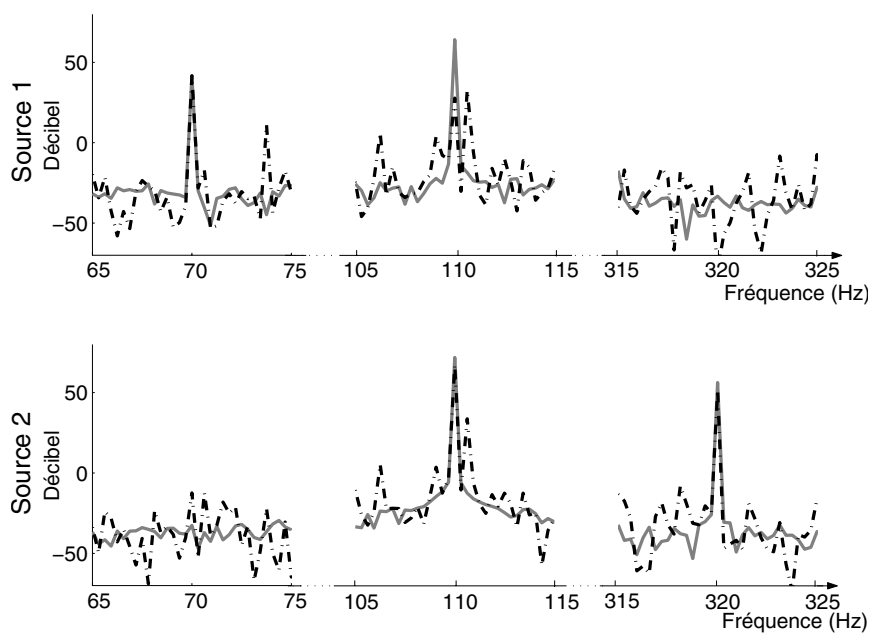

(b)

Fig. 8. Sources restituées à partir de la voie : (a) V4 et (b) V8. (noir) sources réelles, (gris pointillés) sources calculées par inversion.

\subsubsection{Restitution des sources}

Cette section illustre des restitutions dans le cas d'une position optimisée et d'une position non optimisée. Les résultats de la restitution sont présentées en figure 8 .

Dans une zone mal conditionnée, la restitution nous révèle une erreur de restitution pour la fréquence de $110 \mathrm{~Hz}$ de l'ordre de $38 \mathrm{~dB}$, tandis que dans une zone bien conditionnée, la restitution est parfaite. Revenons sur la restitution à partir de la mauvaise zone et visualisons les cartes de conditionnement obtenues par l'approche numérique (Fig. 4) de chacune des fréquences. Les fréquences $70 \mathrm{~Hz}$ et $320 \mathrm{~Hz}$ sont sujettes à une bonne restitution, contrairement à la fréquence $110 \mathrm{~Hz}$, c'est pourquoi l'inversion est délicate pour cette fréquence. Et ceci sera d'autant plus vrai que les signaux capteurs ou les fonctions de transmissibilité sont contaminés par du bruit.

\section{Conclusion}

Cet article présente deux approches dans le cadre de la restitution énergétique d'une fréquence. Ces approches sont basées sur la détermination de zones de placement optimal des capteurs à travers une carte de la structure appelée carte de conditionnement. La première approche est une approche numérique, elle détermine point par point la carte de conditionnement. Cette approche offre la possibilité d'obtenir une carte de conditionnement précise mais nécessite la connaissance d'un modèle numérique. La deuxième approche par analyse modale expérimentale permet de s'affranchir du modèle numérique mais nécessite une détermination des modes dominants pour chacune des fréquences à restituer. Par ailleurs cette approche n'est viable que si le nombre de sources est limité mais aussi un nombre de modes dominants relativement petit pour réussir à interpréter les relations présentées dans cet article. Une analyse sur une structure plus complexe suit cette étude. Cette structure sera un carter constitué d'un arbre tournant, il s'agit d'un élément d'un banc qui a été réalisé dans le cadre d'un projet État-Région nommé «SURVIB ». Ce banc permettra des analyses de fatigue, de suivi d'endommagement, et le développement de méthodes de restitution de sources.

Remerciements. Les auteurs tiennent à remercier la région Champagne-Ardenne et l'État pour leur aide financière à travers le projet État-Région SURVIB.

\section{Annexe}

Cette annexe propose la démonstration permettant d'obtenir la relation utile à la détermination du positionnement optimal des sorties sur une structure.

\section{Matrice de transmissibilité}

Dans le cadre général, le terme général de la matrice de transmissibilité s'écrit à l'aide de l'équation (27)

$$
\begin{array}{r}
t_{x_{j} \rightarrow y_{i}}=\mathbf{T}_{i, j}(\omega)=\frac{\sum_{k=1}^{M} \frac{\phi_{k}\left(x_{j}\right) \phi_{k}\left(y_{i}\right)}{-\omega^{2} M_{k}+\mathbf{i} \omega C_{k}+K_{k}}}{\sum_{k=1}^{M} \frac{\phi_{k}\left(x_{j}\right) \phi_{k}\left(x_{j}\right)}{-\omega^{2} M_{k}+\mathbf{i} \omega C_{k}+K_{k}}} \\
\text { avec } i, j=1, \ldots, p
\end{array}
$$

$x_{i}$ et $y_{i}$ désignent respectivement les entrées et les sorties. $M_{k}, C_{k}$ et $K_{k}$ sont les $k^{\text {ème }}$ composantes de la diagonale des matrices de masse, de raideur, et d'amortissement exprimées dans la base modale. $\mathbf{i}$ est le nombre complexe tel que $\mathbf{i}^{2}=-1 . \phi_{i}$ est le $i^{\text {ème }}$ vecteur propre. Pour alléger l'écriture, la notation suivante est introduite :

$$
D_{k}=-\omega^{2} M_{k}+\mathbf{i} \omega C_{k}+K_{k}
$$


En tenant compte de la notation introduite, le déterminant de la matrice de mélange est :

$$
\begin{aligned}
& \operatorname{det}(\mathbf{T}(\omega))=\frac{1}{\prod_{j=1}^{p} \sum_{k=1}^{M} \frac{\phi_{k}\left(x_{j}\right) \phi_{k}\left(x_{j}\right)}{D_{k}}} \\
& \times\left|\begin{array}{ll}
\sum_{k=1}^{M} \frac{\phi_{k}\left(x_{1}\right) \phi_{k}\left(y_{1}\right)}{D_{k}} \sum_{k=1}^{M} \frac{\phi_{k}\left(x_{2}\right) \phi_{k}\left(y_{1}\right)}{D_{k}} \ldots \sum_{k=1}^{M} \frac{\phi_{k}\left(x_{p}\right) \phi_{k}\left(y_{1}\right)}{D_{k}} \\
\sum_{k=1}^{M} \frac{\phi_{k}\left(x_{1}\right) \phi_{k}\left(y_{2}\right)}{D_{k}} \sum_{k=1}^{M} \frac{\phi_{k}\left(x_{2}\right) \phi_{k}\left(y_{2}\right)}{D_{k}} \ldots \\
\vdots \\
\sum_{k=1}^{M} \frac{\phi_{k}\left(x_{1}\right) \phi_{k}\left(y_{p}\right)}{D_{k}} \\
=\frac{\prod_{k=1}^{M} \frac{\phi_{k}\left(x_{p}\right) \phi_{k}\left(y_{p}\right)}{D_{k}}}{\prod_{j=1}^{p} \sum_{k=1}^{M} \frac{\phi_{k}\left(x_{j}\right) \phi_{k}\left(x_{j}\right)}{D_{k}} \cdot \operatorname{det}\left(\mathbf{T}^{\prime}(\omega)\right)}
\end{array}\right|
\end{aligned}
$$

Pour avoir un système inversible la relation doit être nonnulle, or

$$
\frac{1}{\prod_{j} \sum_{k} \frac{\phi_{k}\left(x_{j}\right) \phi_{k}\left(x_{j}\right)}{D_{k}}} \neq 0
$$

dans ce cas le déterminant de $\mathbf{T}^{\prime}(\omega)$ doit être non nul. Sa valeur dépend des entrées, des sorties et de la pulsation :

$$
\begin{aligned}
& \operatorname{det}\left(\mathbf{T}^{\prime}(\omega)\right)= \\
& \left|\begin{array}{ccc}
\sum_{k=1}^{M} \frac{\phi_{k}\left(x_{1}\right) \phi_{k}\left(y_{1}\right)}{D_{k}} \sum_{k=1}^{M} \frac{\phi_{k}\left(x_{2}\right) \phi_{k}\left(y_{1}\right)}{D_{i}} & \ldots \sum_{k=1}^{M} \frac{\phi_{k}\left(x_{p}\right) \phi_{k}\left(y_{1}\right)}{D_{k}} \\
\sum_{k=1}^{M} \frac{\phi_{k}\left(x_{1}\right) \phi_{k}\left(y_{2}\right)}{D_{k}} \sum_{k=1}^{M} \frac{\phi_{k}\left(x_{2}\right) \phi_{k}\left(y_{2}\right)}{D_{i}} & \ldots & \vdots \\
\vdots & \ddots & \vdots \\
\sum_{k=1}^{M} \frac{\phi_{k}\left(x_{1}\right) \phi_{k}\left(y_{p}\right)}{D_{k}} & & \sum_{k=1}^{M} \frac{\phi_{k}\left(x_{p}\right) \phi_{k}\left(y_{p}\right)}{D_{k}}
\end{array}\right| \neq 0
\end{aligned}
$$

Dans le but d'expliciter la relation, plusieurs étapes sont nécessaires.

\section{Système $M$ modes, 2 entrées, 2 sorties $(M, p=2)$}

La relation est ici déterminée dans le cas de deux entrées, deux sorties et $m$ modes dominants. Dans ce cadre, le déterminant de la matrice de transmissibilité s'exprime simplement :

$$
\begin{aligned}
& \operatorname{det}\left(\mathbf{T}^{\prime}\right)=\left|\begin{array}{l}
\sum_{k=1}^{M} \frac{\phi_{k}\left(x_{1}\right) \phi_{k}\left(y_{1}\right)}{D_{k}} \sum_{k=1}^{M} \frac{\phi_{k}\left(x_{2}\right) \phi_{k}\left(y_{1}\right)}{D_{k}} \\
\sum_{k=1}^{M} \frac{\phi_{k}\left(x_{1}\right) \phi_{k}\left(y_{2}\right)}{D_{k}} \sum_{k=1}^{M} \frac{\phi_{k}\left(x_{2}\right) \phi_{k}\left(y_{2}\right)}{D_{k}}
\end{array}\right| \\
& \operatorname{det}\left(\mathbf{T}^{\prime}\right)=-\sum_{k=1}^{M} \frac{\phi_{k}\left(x_{2}\right) \phi_{k}\left(y_{1}\right)}{D_{k}} \cdot \sum_{k=1}^{M} \frac{\phi_{k}\left(x_{1}\right) \phi_{k}\left(y_{2}\right)}{D_{k}} \\
& +\sum_{k=1}^{M} \frac{\phi_{k}\left(x_{2}\right) \phi_{k}\left(y_{2}\right)}{D_{k}} \cdot \sum_{k=1}^{M} \frac{\phi_{k}\left(x_{1}\right) \phi_{k}\left(y_{1}\right)}{D_{k}}
\end{aligned}
$$

Après développement, d'une part les termes en $D_{k}^{2}$ s'annulent, d'autre part le développement des termes croisés $D_{j} D_{k}, j<k$, permet d'obtenir la relation équation (31)

$$
\begin{aligned}
& \operatorname{det}\left(\mathbf{T}^{\prime}\right)=\sum_{j, k=1 ; j<k}^{M} \\
& \times \frac{\left[\phi_{j}\left(x_{1}\right) \phi_{k}\left(x_{2}\right)-\phi_{j}\left(x_{2}\right) \phi_{k}\left(x_{1}\right)\right]\left[\phi_{j}\left(y_{1}\right) \phi_{k}\left(y_{2}\right)-\phi_{j}\left(y_{2}\right) \phi_{k}\left(y_{1}\right)\right]}{D_{j} D_{k}}
\end{aligned}
$$

Pour un système MIMO, 2 entrées, 2 sorties et $M$ modes dominants la relation à vérifier est l'équation (32) :

$$
\begin{aligned}
\mid \sum_{j, k=1 ; j<k}^{M} \frac{1}{D_{j} D_{k}} & {\left[\phi_{j}\left(x_{1}\right) \phi_{k}\left(x_{2}\right)-\phi_{j}\left(x_{2}\right) \phi_{k}\left(x_{1}\right)\right] } \\
\times & {\left[\phi_{j}\left(y_{1}\right) \phi_{k}\left(y_{2}\right)-\phi_{j}\left(y_{2}\right) \phi_{k}\left(y_{1}\right)\right] \mid \neq 0 }
\end{aligned}
$$

\section{Système $M$ modes, 3 entrées, 3 sorties $(M, p=3)$}

Le déterminant pour un système 3 entrées, 3 sorties s'écrit suivant l'équation :

$\operatorname{det}\left(\mathbf{T}^{\prime}\right)=$

$$
\left|\begin{array}{l}
\sum_{k=1}^{M} \frac{\phi_{k}\left(x_{1}\right) \phi_{k}\left(y_{1}\right)}{D_{k}} \sum_{k=1}^{M} \frac{\phi_{k}\left(x_{2}\right) \phi_{k}\left(y_{1}\right)}{D_{k}} \sum_{i=1}^{M} \frac{\phi_{k}\left(x_{3}\right) \phi_{k}\left(y_{1}\right)}{D_{k}} \\
\sum_{k=1}^{M} \frac{\phi_{k}\left(x_{1}\right) \phi_{k}\left(y_{2}\right)}{D_{k}} \sum_{k=1}^{M} \frac{\phi_{k}\left(x_{2}\right) \phi_{k}\left(y_{2}\right)}{D_{k}} \sum_{i=1}^{M} \frac{\phi_{k}\left(x_{3}\right) \phi_{k}\left(y_{2}\right)}{D_{k}} \\
\sum_{k=1}^{M} \frac{\phi_{k}\left(x_{1}\right) \phi_{k}\left(y_{3}\right)}{D_{k}} \sum_{k=1}^{M} \frac{\phi_{k}\left(x_{2}\right) \phi_{k}\left(y_{3}\right)}{D_{k}} \sum_{i=1}^{M} \frac{\phi_{k}\left(x_{3}\right) \phi_{k}\left(y_{3}\right)}{D_{k}}
\end{array}\right|
$$




$$
\begin{aligned}
& \operatorname{det}\left(\mathbf{T}^{\prime}\right)=T_{1}+T_{2}=\sum_{k, k, l ; k<l} t_{1}(k, k, l)+\sum_{k, q, l ; k<l<q} t_{2}(k, q, l) \\
& t_{1}=\frac{\phi_{k}\left(x_{3}\right) \phi_{k}\left(y_{1}\right)}{D_{k} D_{k} D_{l}} \cdot\left[\phi_{k}\left(x_{1}\right) \phi_{l}\left(x_{2}\right)-\phi_{k}\left(x_{2}\right) \phi_{l}\left(x_{1}\right)\right]\left[\phi_{k}\left(y_{2}\right) \phi_{l}\left(y_{3}\right)-\phi_{k}\left(y_{3}\right) \phi_{l}\left(y_{2}\right)\right] \\
& -\frac{\phi_{k}\left(x_{3}\right) \phi_{k}\left(y_{2}\right)}{D_{k} D_{k} D_{l}} \cdot\left[\phi_{k}\left(x_{1}\right) \phi_{l}\left(x_{2}\right)-\phi_{k}\left(x_{2}\right) \phi_{l}\left(x_{1}\right)\right]\left[\phi_{k}\left(y_{1}\right) \phi_{l}\left(y_{3}\right)-\phi_{k}\left(y_{3}\right) \phi_{l}\left(y_{1}\right)\right] \\
& +\frac{\phi_{k}\left(x_{3}\right) \phi_{k}\left(y_{3}\right)}{D_{k} D_{k} D_{l}} \cdot\left[\phi_{k}\left(x_{1}\right) \phi_{l}\left(x_{2}\right)-\phi_{k}\left(x_{2}\right) \phi_{l}\left(x_{1}\right)\right]\left[\phi_{k}\left(y_{1}\right) \phi_{l}\left(y_{2}\right)-\phi_{k}\left(y_{2}\right) \phi_{l}\left(y_{1}\right)\right] \\
& =\phi_{k}\left(x_{3}\right) A\left(D_{k} D_{k} D_{l}\right)^{-1}\left[\phi_{k}\left(y_{1}\right) \phi_{k}\left(y_{2}\right) \phi_{l}\left(y_{3}\right)-\phi_{k}\left(y_{1}\right) \phi_{k}\left(y_{3}\right) \phi_{l}\left(y_{2}\right)-\phi_{k}\left(y_{2}\right) \phi_{k}\left(y_{1}\right) \phi_{l}\left(y_{3}\right)\right. \\
& \left.+\phi_{k}\left(y_{2}\right) \phi_{k}\left(y_{3}\right) \phi_{l}\left(y_{1}\right)+\phi_{k}\left(y_{3}\right) \phi_{k}\left(y_{1}\right) \phi_{l}\left(y_{2}\right)-\phi_{k}\left(y_{3}\right) \phi_{k}\left(y_{2}\right) \phi_{l}\left(y_{1}\right)\right] \\
& =0 \\
& \text { avec } A=\left[\phi_{k}\left(x_{1}\right) \phi_{l}\left(x_{2}\right)-\phi_{k}\left(x_{2}\right) \phi_{l}\left(x_{1}\right)\right] \\
& t_{2}=\frac{\phi_{k}\left(x_{3}\right) \phi_{k}\left(y_{1}\right)}{D_{k}} \cdot \frac{1}{D_{l} D_{q}} \cdot\left[\phi_{l}\left(x_{1}\right) \phi_{q}\left(x_{2}\right)-\phi_{l}\left(x_{2}\right) \phi_{q}\left(x_{1}\right)\right]\left[\phi_{l}\left(y_{2}\right) \phi_{q}\left(y_{3}\right)-\phi_{l}\left(y_{3}\right) \phi_{q}\left(y_{2}\right)\right] \\
& +\frac{\phi_{l}\left(x_{3}\right) \phi_{l}\left(y_{1}\right)}{D_{l}} \cdot \frac{1}{D_{k} D_{q}} \cdot\left[\phi_{k}\left(x_{1}\right) \phi_{q}\left(x_{2}\right)-\phi_{k}\left(x_{2}\right) \phi_{q}\left(x_{1}\right)\right]\left[\phi_{k}\left(y_{2}\right) \phi_{q}\left(y_{3}\right)-\phi_{k}\left(y_{3}\right) \phi_{q}\left(y_{2}\right)\right] \\
& +\frac{\phi_{q}\left(x_{3}\right) \phi_{q}\left(y_{1}\right)}{D_{q}} \cdot \frac{1}{D_{k} D_{l}} \cdot\left[\phi_{k}\left(x_{1}\right) \phi_{l}\left(x_{2}\right)-\phi_{k}\left(x_{2}\right) \phi_{l}\left(x_{1}\right)\right]\left[\phi_{k}\left(y_{2}\right) \phi_{l}\left(y_{3}\right)-\phi_{k}\left(y_{3}\right) \phi_{l}\left(y_{2}\right)\right] \\
& -\frac{\phi_{k}\left(x_{3}\right) \phi_{k}\left(y_{2}\right)}{D_{k}} \cdot \frac{1}{D_{l} D_{q}} \cdot\left[\phi_{l}\left(x_{1}\right) \phi_{q}\left(x_{2}\right)-\phi_{l}\left(x_{2}\right) \phi_{q}\left(x_{1}\right)\right]\left[\phi_{l}\left(y_{1}\right) \phi_{q}\left(y_{3}\right)-\phi_{l}\left(y_{3}\right) \phi_{q}\left(y_{1}\right)\right] \\
& -\frac{\phi_{l}\left(x_{3}\right) \phi_{l}\left(y_{2}\right)}{D_{l}} \cdot \frac{1}{D_{k} D_{q}} \cdot\left[\phi_{k}\left(x_{1}\right) \phi_{q}\left(x_{2}\right)-\phi_{k}\left(x_{2}\right) \phi_{q}\left(x_{1}\right)\right]\left[\phi_{k}\left(y_{1}\right) \phi_{q}\left(y_{3}\right)-\phi_{k}\left(y_{3}\right) \phi_{q}\left(y_{1}\right)\right] \\
& -\frac{\phi_{q}\left(x_{3}\right) \phi_{q}\left(y_{2}\right)}{D_{q}} \cdot \frac{1}{D_{k} D_{l}} \cdot\left[\phi_{k}\left(x_{1}\right) \phi_{l}\left(x_{2}\right)-\phi_{k}\left(x_{2}\right) \phi_{l}\left(x_{1}\right)\right]\left[\phi_{k}\left(y_{1}\right) \phi_{l}\left(y_{3}\right)-\phi_{k}\left(y_{3}\right) \phi_{l}\left(y_{1}\right)\right] \\
& +\frac{\phi_{k}\left(x_{3}\right) \phi_{k}\left(y_{3}\right)}{D_{k}} \cdot \frac{1}{D_{l} D_{q}} \cdot\left[\phi_{l}\left(x_{1}\right) \phi_{q}\left(x_{2}\right)-\phi_{l}\left(x_{2}\right) \phi_{q}\left(x_{1}\right)\right]\left[\phi_{l}\left(y_{2}\right) \phi_{q}\left(y_{1}\right)-\phi_{l}\left(y_{1}\right) \phi_{q}\left(y_{2}\right)\right] \\
& +\frac{\phi_{l}\left(x_{3}\right) \phi_{l}\left(y_{3}\right)}{D_{l}} \cdot \frac{1}{D_{k} D_{q}} \cdot\left[\phi_{k}\left(x_{1}\right) \phi_{q}\left(x_{2}\right)-\phi_{k}\left(x_{2}\right) \phi_{q}\left(x_{1}\right)\right]\left[\phi_{k}\left(y_{2}\right) \phi_{q}\left(y_{1}\right)-\phi_{k}\left(y_{1}\right) \phi_{q}\left(y_{2}\right)\right] \\
& +\frac{\phi_{q}\left(x_{3}\right) \phi_{q}\left(y_{3}\right)}{D_{q}} \cdot \frac{1}{D_{k} D_{l}} \cdot\left[\phi_{k}\left(x_{1}\right) \phi_{l}\left(x_{2}\right)-\phi_{k}\left(x_{2}\right) \phi_{l}\left(x_{1}\right)\right]\left[\phi_{k}\left(y_{2}\right) \phi_{l}\left(y_{1}\right)-\phi_{k}\left(y_{1}\right) \phi_{l}\left(y_{2}\right)\right]
\end{aligned}
$$

Le calcul du déterminant est réalisé par le développement à partir de la troisième colonne :

$$
\begin{aligned}
& \operatorname{det}\left(\mathbf{T}^{\prime}\right)=\sum_{k=1}^{M} \frac{\phi_{k}\left(x_{3}\right) \phi_{k}\left(y_{1}\right)}{D_{k}} \cdot \sum_{j, k=1 ; j<k}^{M} \\
& \times \frac{\left[\phi_{j}\left(x_{1}\right) \phi_{k}\left(x_{2}\right)-\phi_{j}\left(x_{2}\right) \phi_{k}\left(x_{1}\right)\right]\left[\phi_{j}\left(y_{2}\right) \phi_{k}\left(y_{3}\right)-\phi_{j}\left(y_{3}\right) \phi_{k}\left(y_{2}\right)\right]}{D_{j} D_{k}} \\
& -\sum_{k=1}^{M} \frac{\phi_{k}\left(x_{3}\right) \phi_{k}\left(y_{2}\right)}{D k} \cdot \sum_{j, k=1 ; j<k}^{M} \\
& \times \frac{\left[\phi_{j}\left(x_{1}\right) \phi_{k}\left(x_{2}\right)-\phi_{j}\left(x_{2}\right) \phi_{k}\left(x_{1}\right)\right]\left[\phi_{j}\left(y_{1}\right) \phi_{k}\left(y_{3}\right)-\phi_{j}\left(y_{3}\right) \phi_{k}\left(y_{1}\right)\right]}{D_{j} D_{k}} \\
& \left.+\sum_{k=1}^{M} \frac{\phi_{k}\left(x_{3}\right) \phi_{k}\left(y_{3}\right)}{D_{k}} \cdot \sum_{j, k=1 ; j<k}^{M} \phi_{j}\left(x_{2}\right) \phi_{k}\left(x_{1}\right)\right]\left[\phi_{j}\left(y_{1}\right) \phi_{k}\left(y_{2}\right)-\phi_{j}\left(y_{2}\right) \phi_{k}\left(y_{1}\right)\right] \\
& D_{j} D_{k}
\end{aligned}
$$

Le développement de l'expression précédente est réalisé en deux étapes, le développement des termes en $D_{k} D_{k} D_{l}$, noté $T_{1}=\sum_{k, k, l ; k \neq l} t 1(k, k, l), k<l$ et des termes en $D_{k} D_{l} D_{q}, k<l<q$, noté $T_{2}=$ $\sum_{k, q, l ; k<l<q} t_{2}(k, q, l)$ voir équation (33) ci-dessus.
Une factorisation est possible, révélant le terme représentant les vecteurs propres des sorties, noté $\Phi(y, k, l, q)$, et les vecteurs propres des entrées, noté $\Phi(x, k, l, q)$ :

$$
t_{2}=\frac{1}{D_{k} D_{q} D_{l}} \Phi(x, k, l, q) \Phi(y, k, l, q)
$$

avec

$$
\begin{aligned}
& \Phi(y, k, l, q)=\sum_{i=1,2,3 ; j=0,1}(-1)^{j} \phi_{k}\left(y_{i+2 j}\right) \phi_{l}\left(y_{i+1}\right) \\
& \times \phi_{q}\left(y_{i+2-2 j}\right), i+3 k^{\prime} \equiv i[3], k^{\prime} \in \mathbb{N} \\
& \Phi(x, k, l, q)=\sum_{i=1,2,3 ; j=0,1}(-1)^{j} \phi_{k}\left(x_{i+2 j}\right) \phi_{l}\left(x_{i+1}\right) \\
& \times \phi_{q}\left(x_{i+2-2 j}\right), i+3 k^{\prime} \equiv i[3], k^{\prime} \in \mathbb{N}
\end{aligned}
$$

Le déterminant de $\mathbf{T}^{\prime}$ se simplifie donc suivant l'équation (5) :

$$
\operatorname{det}\left(\mathbf{T}^{\prime}\right)=T_{1}+T_{2}=T_{2}
$$




$$
\begin{array}{|l}
\left|\sum_{k_{1}, k_{2}, \ldots, k_{p}=1}^{M} \frac{1}{D_{k_{1} \ldots D_{k_{p}}}} \Phi\left(x, k_{1}, \ldots, k_{p}\right) . \Phi\left(y, k_{1}, \ldots, k_{p}\right)\right| \neq 0, \quad k_{1}<k_{2}<\ldots<k_{p}, \forall i, k_{i} \in \mathbb{N} \\
\text { où } \Phi\left(x, k_{1}, \ldots, k_{p}\right)=\sum_{\substack{i=1 \text { à } p \\
j=0,1}}(-1)^{j} \phi_{k_{1}}\left(x_{i+(p-1) j}\right) \phi_{k_{2}}\left(x_{i+1+(p-3) j}\right) \ldots \phi_{k_{p}}\left(x_{i+2+(p-2 p+1) j}\right) \\
\text { où } \Phi\left(y, k_{1}, \ldots, k_{p}\right)=\sum_{\substack{i=1 \text { à } p \\
j=0,1}}(-1)^{j} \phi_{k_{1}}\left(y_{i+(p-1) j}\right) \phi_{k_{2}}\left(y_{i+1+(p-3) j}\right) \ldots \phi_{k_{p}}\left(y_{i+2+(p-2 p+1) j}\right)
\end{array}
$$

Pour un système MIMO, 3 entrées, 3 sorties et $M$ modes dominants la relation à vérifier est l'équation (34) :

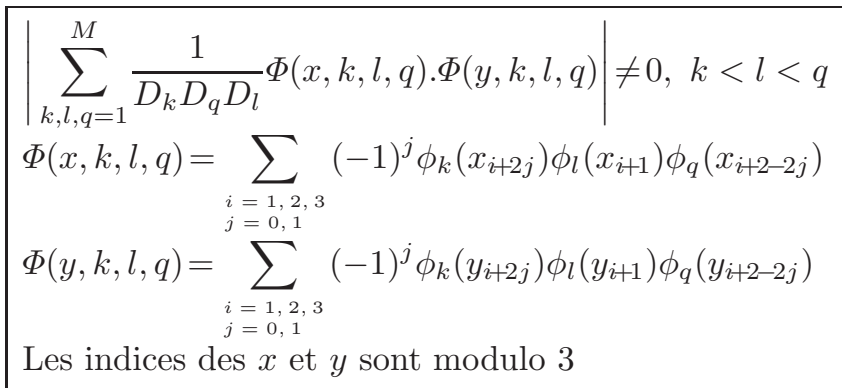

\section{Système $M$ modes, $p$ entrées, $p$ sorties $(M, p \geq 3)$}

La relation équation (34) peut être généralisée à un système MIMO $p$ entrées, $p$ sorties et $m$ modes dominants. La relation est obtenue à l'aide d'une récurrence et un développement sur la $p^{\text {ième }}$ colonne. Le détail du calcul, étant fastidieux, seul le résultat apparaît dans l'équation (35) :

$$
\text { voir équation (35) ci-dessus. }
$$

\section{Références}

[1] M. Misiti, Y. Misiti, G. Oppenheimer, Les ondelettes et leurs applications, Paris, Hermès Science Publications, Lavoisier, 2003

[2] M. Bonnet (coord.), Problèmes inverses : De l'expérimentation à la modélisation, Paris, Arago Édition, 1999

[3] J. Morel, Surveillance vibratoire et maintenance prédictive, Les techniques de l'ingénieur, 1992

[4] N.G. Nikolaou, I.A. Antoniadis, Demodulation of vibration signals generated by defects in rolling element bearings using complex shifted morlet wavelets, Mechanical Systems and Signal Processing 16 (2002) 677-694

[5] P.D. McFadden, J.D. Smith, Vibration monitoring of rolling element bearings by the high-frequency resonance technique-a review, Tribology International 17 (1984) $3-10$

[6] J.P. Dron, F. Bolaers, L. Rasolofondraibe, Influence of the de-noising method by spectral subtraction on the diagnosis of rolling bearings, Eur. J. mechanical and environmental engineering 48 (2003) 3-12

[7] F. Bolaers, Contribution à l'étude et au développement d'un système intégré de suivi vibratoire de l'endommagement de composants mécaniques sur machines tournantes, thèse, Université de Reims, 2002

[8] N. Tandon, A. Choudhury, A review of vibration and acoustic measurement methods for the detection of defects in rolling element bearings, Tribology International 32 (1999) 469-480

[9] F. Bogard, K. Debray, Y.Q. Guo, Determination of sensor positions for predictive maintenance of revolving machines, Int. J. Solids and Structures 39 (2002) 3159-3173

[10] X. Chiementin, F. Bolaers, L. Rasolofondraibe, J.-P. Dron, Systematic restitution method of vibratory sources for a mechanical system, $5^{\mathrm{e}}$ congrès international sur les méthodes de surveillance et techniques acoustiques et vibratoires, CETIM-Senlis, octobre 2004

[11] C. Pezerat, Méthode d'identification des efforts appliqués sur une structure vibrante, par résolution et régularisation du problème inverse, thèse, INSA Lyon, 1996

[12] R. Dib, Caractérisation vibratoire des machines industrielles par des méthodes pseudo inverses : méthodologie, instrumentation et expérimentation, thèse, Université de Technologie Compiègne, 2002

[13] Q. Leclere, Étude et développement de la mesure indirecte d'efforts : Application à l'identification des sources internes d'un moteur Diesel, thèse, Insa de Lyon, 2003

[14] N. Totaro, Caractérisation de sources aérodynamiques et sous-structuration pour la méthode SEA, thèse, INSA de Lyon, 2004

[15] X. Chiementin, F. Bolaers, L. Rasolofondraibe, J.-P. Dron, Optimisation des problèmes inverses pour la caractérisation des sources vibratoires, Premier congrès international Conception et Modélisation des Systèmes Mécaniques, Hammamet, Tunisie, Mars 2005

[16] J. Hadamard, Lectures on Cauchy's Problem in Linear Partial Differential Equations, Yale University Press, 1923

[17] A. Zhang, Détermination des forces appliquées aux structures industrielles par des méthodes inverses, thèse, Université Paul Sabatier, Toulouse, 1998

[18] A. Tikhonov, V. Arsénine, Méthodes de résolution des problèmes mal posés, Moscou, 1976

[19] M. Géradin, D. Rixen, Theory and Application to Structural Dynamics, John Wiley, Wiley and Sons, 1993 